VoL. 54 (1996) [131-146]

\title{
NEW TELYAKOVSKII-TYPE ESTIMATES VIA THE BOOLEAN SUM APPROACH
}

\author{
Jia-ding CaO and Heinz H. Gonska
}

\begin{abstract}
In the present note the magnitude of constants in Telyakovskii-type theorems is investigated. Our general approach to construct the linear operators yielding good constants is the one via Boolean sums. Explicit values for the constants in question are given for general convolution-type operators; the classical Fejér-Korovkin kernel is then used as an example for which one obtains rather small values. Furthermore, also an asymptotic assertion is derived which indicates the room left for improvement of the main results. This leads to a natural conjecture concluding this article.
\end{abstract}

\section{INTRODUCTION}

Let $\mathbb{N}=\{1,2, \ldots\}$ be the set of natural numbers. For $f \in C[a, b]$ (real-valued and continuous functions on the compact interval $[a, b])$, let $\|f\|:=\max \{|f(t)|: a \leqslant t \leqslant b\}$ denote the Čebyšev norm of $f$. For $k \in \mathbb{N}_{0}=\mathbb{N} \cup\{0\}$, by $C^{k}[a, b]$ we denote the space of $k$-fold continuously differentiable functions; $\pi_{n}$ will be the set of algebraic polynomials of degree $\leqslant n$. For $f \in C[a, b]$, the modulus of continuity of $f$ is defined by

$$
\omega(f, \delta):=\sup \left\{\left|f\left(x_{1}\right)-f\left(x_{2}\right)\right|:\left|x_{1}-x_{2}\right| \leqslant \delta\right\}, \quad 0 \leqslant \delta \leqslant b-a .
$$

In his well-known paper [17], Telyakovskii proved the following

Theorem A. For $n \in \mathbb{N}$ and $f \in C[-1,1]$ there exists $P_{n}(f, \cdot) \in \pi_{n}$ such that

$$
\left|f(x)-P_{n}(f, x)\right| \leqslant c \cdot \omega\left(f, \frac{\sqrt{1-x^{2}}}{n}\right) \text { for all }|x| \leqslant 1 \text {, }
$$

where the constant $c$ is independent of $f, n$ and $x$.

In our earlier paper [3] we studied general conditions under which certain Boolean sums of linear operators satisfy Telyakovskiî-type estimates. See [3] and the references

\section{Received 26 September 1995}

The authors would like to express their gratitude to Eva Müller-Faust (European Business School), Rita Hülsbusch (University of Duisburg), and to Dr. Jon Sevy (Allentown College, U.S.A.) for their technical assistance during preparation of this work.

Copyright Clearance Centre, Inc. Serial-fee code: 0004-9729/96 \$A2.00+0.00. 
there for more information concerning this theorem. In the present note we continue our research on these types of inequalities. The central aim here is an investigation of the magnitude of the constant $c$ in Theorem A (Section 2). Earlier papers in which explicit values of the constant $c$ were found are, for example, those by Lehnhoff [12] and Lupas [13], however, the constants given here are much smaller than theirs and thus bring us closer to the solution of the underlying extremal problem. In Section 3 we derive an asymptotic assertion indicating the room left for improvement of the main results in Section 2. More specifically, we show there that a certain asymptotic constant equals $1 / 2$ which, in a very natural fashion, leads to a conjecture concluding that section.

\section{On the constant in Theorem A}

For $n \in \mathbb{N}$, let the even trigonometric kernel $K_{m(n)}$ be given by

$$
K_{m(n)}(v):=\frac{1}{2}+\sum_{k=1}^{m(n)} \rho_{k, m(n)} \cos k v,
$$

where $m$ is a function increasing with $n$.

Let $f \in C[-1,1]$ and $x \in[-1,1]$. The convolution-type operators $G_{m(n)}$ defined by

$$
G_{m(n)}(f, x):=\frac{1}{\pi} \int_{-\pi}^{\pi} f(\cos (v+\arccos x)) \cdot K_{m(n)}(v) d v
$$

were investigated in a series of papers by Pičugov [14], Lehnhoff $[11,12]$, and the present authors (see, for example, [4] and the references cited there).

We have $(1 / \pi) \int_{-\pi}^{\pi} K_{m(n)}(v) d v=1$, and $G_{m(n)}(f, \cdot)$ is an algebraic polynomial of degree $m(n)$.

The main focus in our previous papers was on the order of approximation by positive operators $G_{m(n)}$ and certain Boolean sum modifications of them. Let us recall the following notation and definitions.

For $f \in C[a, b]$, by $L f$ we denote the linear function interpolating $f$ at $a$ and $b$, that is

$$
L(f, x):=\frac{f(b)(x-a)+f(a)(b-x)}{b-a} .
$$

If $A: C[a, b] \rightarrow C[a, b]$ is a linear operator, the Boolean $\operatorname{sum} L \oplus A$ of $L$ and $A$ is given by $A^{+}:=L \oplus A=L+A-L \circ A$, or more explicitly,

$$
A^{+}(f, x)=A(f, x)+(b-a)^{-1}\{(x-a)[f(b)-A(f, b)]+(b-x)[f(a)-A(f, a)]\} .
$$


In the sequel we shall be dealing with the operators

$$
\begin{aligned}
G_{m(n)}^{+}(f, x):=G_{m(n)}(f, x)+ & \frac{1}{2}(x+1)\left[f(1)-G_{m(n)}(f, 1)\right] \\
& +\frac{1}{2}(1-x)\left[f(-1)-G_{m(n)}(f,-1)\right] .
\end{aligned}
$$

We shall need two lemmas taken from papers by Cao (see $[1,2])$.

LEMma 2.1. Let $K_{m(n)}(v) \geqslant 0 \in, v \in \mathbb{R}$. Then the following inequality holds:

$$
2 \cdot \pi^{-1} \int_{-\pi}^{\pi}\left|\sin \frac{1}{2} \xi\right| \cdot K_{m(n)}(\xi) d \xi \leqslant \sqrt{2} \cdot \sqrt{1-\rho_{1, m(n)}} .
$$

Lemma 2.2. If $K_{m(n)}(v) \geqslant 0, v \in \mathbb{R}$, and $|x| \leqslant 1$, then we have

$$
G_{m(n)}(|u-x|, x) \leqslant\left(1-\rho_{1, m(n)}\right) \cdot|x|+\sqrt{2} \cdot \sqrt{1-\rho_{1, m(n)}} \cdot \sqrt{1-x^{2}} .
$$

One further inequality needed will be:

LEMma 2.3. For $n \in \mathbb{N}$, let $K_{m(n)}(v) \geqslant 0, v \in \mathbb{R}$. Then for all $g \in C^{1}[-1,1]$ and all $|x| \leqslant 1$ we have

$$
\left|g(x)-G_{m(n)}^{+}(g, x)\right| \leqslant(2+2 \cdot \sqrt{2}) \cdot \sqrt{1-\rho_{1, m(n)}} \cdot\left\|g^{\prime}\right\| .
$$

If $\rho_{1, m(n)} \geqslant 0$, then the constant $2+2 \cdot \sqrt{2}$ may be replaced by $3+\sqrt{2}$.

Proof: For simplification we write $\rho_{n}:=\rho_{1, m(n)}$. Since $K_{m(n)}(v) \geqslant 0$ and $K_{m(n)} \neq 0$, we have

$$
1-\rho_{n}=\pi^{-1} \int_{-\pi}^{\pi}(1-\cos v) K_{m(n)}(v) d v>0
$$

Let $g \in C^{1}[-1,1]$. Since $|g(x)-g(t)| \leqslant|t-x| \cdot\left\|g^{\prime}\right\|$, and since $G_{m(n)}$ are positive linear operators satisfying $G_{m(n)}(1, x)=1$, we have

$$
\left|g(x)-G_{m(n)}(g, x)\right|=\left|g(x) \cdot G_{m(n)}(1, x)-G_{m(n)}(g, x)\right| \leqslant G_{m(n)}(|t-x|, x) \cdot\left\|g^{\prime}\right\| .
$$

Using Lemma 2.2 we obtain

$$
\left|g(x)-G_{m(n)}(g, x)\right| \leqslant\left[\left(1-\rho_{n}\right) \cdot|x|+\sqrt{2} \sqrt{1-\rho_{n}} \cdot \sqrt{1-x^{2}}\right] \cdot\left\|g^{\prime}\right\| .
$$

Now we apply Lehnhoff's method (see [11] and [12]) and consider three cases: 
CASE (A). $\sqrt{1-\rho_{n}} \leqslant \sqrt{1-x^{2}},-1 \leqslant x \leqslant 1$.

We have

$$
1-\rho_{n} \leqslant \sqrt{1-\rho_{n}} \cdot \sqrt{1-x^{2}}
$$

From (2.6) we obtain

$$
\begin{aligned}
\mid g(x) & -G_{m(n)}^{+}(g, x) \mid \\
\leqslant & \left|g(x)-G_{m(n)}(g, x)\right| \\
& +\frac{1}{2}(x+1) \cdot\left|g(1)-G_{m(n)}(g, 1)\right|+\frac{1}{2}(1-x) \cdot\left|g(-1)-G_{m(n)}(g,-1)\right| \\
\leqslant & {\left[\left(1-\rho_{n}\right) \cdot|x|+\sqrt{2} \cdot \sqrt{1-\rho_{n}} \cdot \sqrt{1-x^{2}}\right] \cdot\left\|g^{\prime}\right\| } \\
& +\frac{1}{2}(x+1) \cdot\left(1-\rho_{n}\right) \cdot\left\|g^{\prime}\right\|+\frac{1}{2}(1-x) \cdot\left(1-\rho_{n}\right) \cdot\left\|g^{\prime}\right\| \\
= & {\left[\left(1-\rho_{n}\right) \cdot|x|+\sqrt{2} \cdot \sqrt{1-\rho_{n}} \cdot \sqrt{1-x^{2}}+\left(1-\rho_{n}\right)\right] \cdot\left\|g^{\prime}\right\| } \\
\leqslant & {\left[\sqrt{1-\rho_{n}} \cdot \sqrt{1-x^{2}}+\sqrt{2} \cdot \sqrt{1-\rho_{n}} \cdot \sqrt{1-x^{2}}+\sqrt{1-\rho_{n}} \cdot \sqrt{1-x^{2}}\right] \cdot\left\|g^{\prime}\right\| } \\
= & (2+\sqrt{2}) \sqrt{1-\rho_{n}} \cdot \sqrt{1-x^{2}} \cdot\left\|g^{\prime}\right\| \cdot
\end{aligned}
$$

CASE (B). $\sqrt{1-x^{2}} \leqslant \sqrt{1-\rho_{n}}, 0 \leqslant x<1$.

From (2.2) we have

$$
\begin{aligned}
g(x)-G_{m(n)}^{+}(g, x)= & {[g(x)-g(1)]-\left[G_{m(n)}(g, x)-G_{m(n)}(g, 1)\right] } \\
& +\frac{1}{2}(1-x)\left\{\left[g(1)-G_{m(n)}(g, 1)\right]-\left[g(-1)-G_{m(n)}(g, 1)\right]\right\} .
\end{aligned}
$$

Now let $I_{m(n)}(x):=\left|G_{m(n)}(g, x)-G_{m(n)}(g, 1)\right|$. Then it follows from (2.6) that

$$
\begin{aligned}
& \left|g(x)-G_{m(n)}^{+}(g, x)\right| \leqslant|g(x)-g(1)|+I_{m(n)}(x) \\
& \quad+\frac{1}{2}(1-x)\left[\left|g(1)-G_{m(n)}(g, 1)\right|+\left|g(-1)-G_{m(n)}(g,-1)\right|\right] \\
& \leqslant(1-x) \cdot\left\|g^{\prime}\right\|+(1-x) \cdot\left(1-\rho_{n}\right) \cdot\left\|g^{\prime}\right\|+I_{m(n)}(x) \\
& \leqslant\left(1-x^{2}\right) \cdot\left\|g^{\prime}\right\|+\left(1-x^{2}\right) \cdot\left(1-\rho_{n}\right) \cdot\left\|g^{\prime}\right\|+I_{m(n)}(x) \\
& \leqslant \sqrt{1-x^{2}} \cdot \sqrt{1-\rho_{n}} \cdot\left\|g^{\prime}\right\|+\left[\sqrt{1-x^{2}} \cdot \sqrt{1-\rho_{n}}\right]^{2} \cdot\left\|g^{\prime}\right\|+I_{m(n)}(x) .
\end{aligned}
$$

Since

$$
1-\rho_{n}=\pi^{-1} \int_{-\pi}^{\pi}(1-\cos v) K_{m(n)}(v) d v \leqslant 2 \cdot \pi^{-1} \int_{-\pi}^{\pi} K_{m(n)}(v) d v=2
$$

we have

$$
\sqrt{1-\rho_{n}} \leqslant \sqrt{2} \text {, and } \sqrt{1-x^{2}} \cdot \sqrt{1-\rho_{n}} \leqslant \sqrt{2}
$$




\section{Hence}

$$
\left|g(x)-G_{m(n)}^{+}(g, x)\right| \leqslant(1+\sqrt{2}) \sqrt{1-\rho_{n}} \cdot \sqrt{1-x^{2}} \cdot\left\|g^{\prime}\right\|+I_{m(n)}(x) .
$$

If $\rho_{n} \geqslant 0$, then $0<1-\rho_{n} \leqslant 1, \sqrt{1-\rho_{n}} \leqslant 1, \sqrt{1-\rho_{n}} \cdot \sqrt{1-x^{2}} \leqslant 1$. Thus in this case we have from (2.8)

$$
\left|g(x)-G_{m(n)}^{+}(g, x)\right| \leqslant 2 \sqrt{1-\rho_{n}} \cdot \sqrt{1-x^{2}} \cdot\left\|g^{\prime}\right\|+I_{m(n)}(x) .
$$

Now we estimate $I_{m(n)}(x)$. We have

$$
\begin{aligned}
I_{m(n)}(x) \leqslant & \pi^{-1} \int_{-\pi}^{\pi}|g(\cos (v+\arccos x))-g(\cos v)| \cdot K_{m(n)}(v) d v \\
\leqslant & \pi^{-1} \cdot\left\|g^{\prime}\right\| \cdot \int_{-\pi}^{\pi}|\cos (v+\arccos x)-\cos v| \cdot K_{m(n)}(v) d v \\
= & \pi^{-1} \cdot\left\|g^{\prime}\right\| \cdot \int_{-\pi}^{\pi}\left|(1-x) \cos v+\sqrt{1-x^{2}} \cdot \sin v\right| \cdot K_{m(n)}(v) d v \\
\leqslant & \pi^{-1} \cdot\left\|g^{\prime}\right\| \cdot\left\{(1-x) \cdot \int_{-\pi}^{\pi}|\cos v| \cdot K_{m(n)}(v) d v\right. \\
& \left.+\sqrt{1-x^{2}} \cdot \int_{-\pi}^{\pi}|\sin v| \cdot K_{m(n)}(v) d v\right\} \\
\leqslant & \pi^{-1} \cdot\left\|g^{\prime}\right\| \cdot\left\{\left(1-x^{2}\right) \cdot \int_{-\pi}^{\pi}|\cos v| \cdot K_{m(n)}(v) d v\right. \\
& \left.+2 \cdot \sqrt{1-x^{2}} \cdot \int_{-\pi}^{\pi}\left|\sin \frac{v}{2}\right| \cdot K_{m(n)}(v) d v\right\}
\end{aligned}
$$

Using Lemma 2.1 we obtain

$$
\begin{aligned}
I_{m(n)}(x) & \leqslant\left(1-x^{2}\right) \cdot \pi^{-1} \cdot \int_{-\pi}^{\pi} K_{m(n)}(v) d v \cdot\left\|g^{\prime}\right\|+\sqrt{2} \cdot \sqrt{1-x^{2}} \cdot \sqrt{1-\rho_{n}} \cdot\left\|g^{\prime}\right\| \\
& =\left(1-x^{2}\right) \cdot\left\|g^{\prime}\right\|+\sqrt{2} \cdot \sqrt{1-x^{2}} \cdot \sqrt{1-\rho_{n}} \cdot\left\|g^{\prime}\right\| \\
& \leqslant\left\{\sqrt{1-x^{2}} \cdot \sqrt{1-\rho_{n}}+\sqrt{2} \cdot \sqrt{1-x^{2}} \cdot \sqrt{1-\rho_{n}}\right\} \cdot\left\|g^{\prime}\right\| \\
& =(1+\sqrt{2}) \sqrt{1-\rho_{n}} \cdot \sqrt{1-x^{2}} \cdot\left\|g^{\prime}\right\| .
\end{aligned}
$$

By combining (2.9) and (2.11) we have

$$
\left|g(x)-G_{m(n)}^{+}(g, x)\right| \leqslant(2+2 \sqrt{2}) \sqrt{1-\rho_{n}} \cdot \sqrt{1-x^{2}} \cdot\left\|g^{\prime}\right\| .
$$


If $\rho_{n} \geqslant 0$, by combining (2.10) and (2.11) we get

$$
\left|g(x)-G_{m(n)}^{+}(g, x)\right| \leqslant(3+\sqrt{2}) \sqrt{1-\rho_{n}} \cdot \sqrt{1-x^{2}} \cdot\left\|g^{\prime}\right\| .
$$

CASE (C). $\sqrt{1-x^{2}} \leqslant \sqrt{1-\rho_{n}},-1<x \leqslant 0$.

From (2.2) it follows that

$$
\begin{gathered}
\left|g(x)-G_{m(n)}^{+}(g, x)\right|=[g(x)-g(-1)]+\left[G_{m(n)}(g,-1)-G_{m(n)}(g, x)\right] \\
+\frac{1}{2}(x+1)\left\{\left[g(-1)-G_{m(n)}(g,-1)\right]+\left[G_{m(n)}(g, 1)-g(1)\right]\right\} .
\end{gathered}
$$

Since $1+x \leqslant 1-x^{2}$ for $-1<x \leqslant 0$, again using a method analogous to the one used in Case (B), we get

$$
\left|g(x)-G_{m(n)}^{+}(g, x)\right| \leqslant(2+2 \sqrt{2}) \sqrt{1-\rho_{n}} \cdot \sqrt{1-x^{2}} \cdot\left\|g^{\prime}\right\|,-1<x \leqslant 0 .
$$

Furthermore, if $\rho_{n} \geqslant 0$, then

$$
\left|g(x)-G_{m(n)}^{+}(g, x)\right| \leqslant(3+\sqrt{2}) \sqrt{1-\rho_{n}} \cdot \sqrt{1-x^{2}} \cdot\left\|g^{\prime}\right\|,-1<x \leqslant 0 .
$$

From (2.2) we get

$$
G_{m(n)}^{+}(g, 1)=g(1) \text { and } G_{m(n)}^{+}(g,-1)=g(-1) .
$$

By combining (2.7) (Case (A)), (2.12) (Case (B)) and (2.14) (Case (C)), we get for $n \geqslant 1$ and $|x| \leqslant 1$,

$$
\left|g(x)-G_{m(n)}^{+}(g, x)\right| \leqslant(2+2 \sqrt{2}) \sqrt{1-\rho_{n}} \cdot \sqrt{1-x^{2}} \cdot\left\|g^{\prime}\right\| .
$$

If $\rho_{n} \geqslant 0$, we combine (2.7), (2.13) and (2.15) to get for $n \geqslant 1$ and $|x| \leqslant 1$,

$$
\left|g(x)-G_{m(n)}^{+}(g, x)\right| \leqslant(3+\sqrt{2}) \sqrt{1-\rho_{n}} \cdot \sqrt{1-x^{2}} \cdot\left\|g^{\prime}\right\| .
$$

In order to get an estimate which is valid for all continuous functions we use the $K$-functional

$$
K(f ; \delta):=\inf \left\{\|f-g\|+\delta \cdot\left\|g^{\prime}\right\|: g \in C^{1}[-1,1]\right\}, \quad f \in C[-1,1], \delta \geqslant 0 .
$$

It is well-known that this functional is related to the least concave majorant $\tilde{\omega}(f, \cdot)$ of the modulus of continuity $\omega(f, \cdot)$ by the equation

$$
K(f ; \delta)=\frac{1}{2} \cdot \widetilde{\omega}(f ; 2 \delta) \text { for all } \delta \geqslant 0
$$

Furthermore, it was shown by Korneičuk [8] that for $\widetilde{\omega}$ and $\omega$ the following is true:

$$
\widetilde{\omega}(f ; \xi \cdot \delta) \leqslant(1+\xi) \cdot \omega(f, \delta) \text { for any } \delta \geqslant 0 \text { and } \xi>0 \text {. }
$$

These facts will be used in the proof of 
TheOREM 2.4. Let $n \in \mathbb{N}, K_{m(n)}(v) \geqslant 0,|x| \leqslant 1$, and $h>0$. Then for $f \in C[-1,1]$ we have

$$
\begin{aligned}
& \left|f(x)-G_{m(n)}^{+}(f, x)\right| \\
& \quad \leqslant\left[2+(2+2 \sqrt{2}) \cdot \sqrt{1-\rho_{1, m(n)}} \cdot h^{-1}\right] \cdot \omega\left(f, h \cdot \sqrt{1-x^{2}}\right) .
\end{aligned}
$$

If $\rho_{1, m(n)} \geqslant 0$, then the factor in front of $\omega(f, \cdot)$ may be replaced by

$$
2+(3+\sqrt{2}) \cdot \sqrt{1-\rho_{1, m(n)}} \cdot h^{-1}
$$

Proof: Observe first that (2.2) implies

$$
\left|G_{m(n)}^{+}(f, x)\right| \leqslant 3 \cdot\|f\| \text { for all } f \in C[-1,1] \text {. }
$$

Again, for simplification we shall write $\rho_{n}:=\rho_{1, m(n)}$.

Now let $g \in C^{1}[-1,1]$ be arbitrary. Then

$$
\begin{aligned}
& \left|f(x)-G_{m(n)}^{+}(f, x)\right| \\
& \leqslant|f(x)-g(x)|+\left|g(x)-G_{m(n)}^{+}(g, x)\right|+\left|G_{m(n)}^{+}(g-f, x)\right| \\
& \leqslant 4 \cdot\|f-g\|+(2+2 \sqrt{2}) \cdot \sqrt{1-\rho_{n}} \cdot \sqrt{1-x^{2}} \cdot\left\|g^{\prime}\right\| \\
& =4 \cdot\left\{\|f-g\|+\frac{2+2 \sqrt{2}}{4} \cdot \sqrt{1-\rho_{n}} \cdot h^{-1} \cdot h \cdot \sqrt{1-x^{2}} \cdot\left\|g^{\prime}\right\|\right\} \text { for any } h>0 .
\end{aligned}
$$

Taking the infimum over $g \in C^{1}[-1,1]$ and using (2.18), (2.19) and (2.20) show

$$
\begin{aligned}
\mid f(x) & -G_{m(n)}^{+}(f, x) \mid \\
& \leqslant 4 \cdot K\left(f, \frac{2+2 \sqrt{2}}{4} \cdot \sqrt{1-\rho_{n}} \cdot h^{-1} \cdot h \cdot \sqrt{1-x^{2}}\right) \\
& =2 \cdot \tilde{\omega}\left(f ;(1+\sqrt{2}) \cdot \sqrt{1-\rho_{n}} \cdot h^{-1} \cdot h \cdot \sqrt{1-x^{2}}\right) \\
& \leqslant 2 \cdot\left(1+(1+\sqrt{2}) \cdot \sqrt{1-\rho_{n}} \cdot h^{-1}\right) \cdot \omega\left(f, h \cdot \sqrt{1-x^{2}}\right) .
\end{aligned}
$$

Furthermore, if $\rho_{n} \geqslant 0$, then in the above, the constant $2+2 \sqrt{2}$ can be replaced by $3+\sqrt{2}$, giving in this case

$$
\left|f(x)-G_{m(n)}^{+}(f, x)\right| \leqslant 2 \cdot\left(1+\frac{3+\sqrt{2}}{2} \sqrt{1-\rho_{n}} \cdot h^{-1}\right) \cdot \omega\left(f, h \cdot \sqrt{1-x^{2}}\right) .
$$


REMARK 2.5. If $\omega(f, \cdot)$ is concave, then

$$
\omega(f, \delta)=\tilde{\omega}(f, \delta) \text { for all } \delta \geqslant 0 .
$$

From the proof of Theorem 2.4 (see (2.23)) it can be seen that in the general case one has, for any $g \in C^{1}[-1,1]$,

$$
\begin{aligned}
& \left|f(x)-G_{m(n)}^{+}(f, x)\right| \\
& \quad \leqslant 2 \cdot \max \left[2,(2+2 \sqrt{2}) \sqrt{1-\rho_{1, m(n)}} \cdot h^{-1}\right] \cdot\left\{\|f-g\|+\frac{h}{2} \sqrt{1-x^{2}}\left\|g^{\prime}\right\|\right\} .
\end{aligned}
$$

Taking the infimum over $g \in C^{1}[-1,1]$ and using (2.18) and (2.19) shows

$$
\begin{aligned}
\mid f(x) & -G_{m(n)}^{+}(f, x) \mid \\
& \leqslant 2 \cdot \max \left[2,(2+2 \sqrt{2}) \cdot \sqrt{1-\rho_{1, m(n)}} \cdot h^{-1}\right] \cdot K\left(f ; \frac{h}{2} \sqrt{1-x^{2}}\right) \\
& =\max \left[2,(2+2 \sqrt{2}) \cdot \sqrt{1-\rho_{1, m(n)}} \cdot h^{-1}\right] \cdot \tilde{\omega}\left(f ; h \cdot \sqrt{1-x^{2}}\right) \\
& =\max \left[2,(2+2 \sqrt{2}) \cdot \sqrt{1-\rho_{1, m(n)}} \cdot h^{-1}\right] \cdot \omega\left(f ; h \cdot \sqrt{1-x^{2}}\right)
\end{aligned}
$$

whereas, for $\rho_{1, m(n)}>0$,

$$
\left|f(x)-G_{m(n)}^{+}(f, x)\right| \leqslant \max \left[2,(3+\sqrt{2}) \cdot \sqrt{1-\rho_{m(n)}} \cdot h^{-1}\right] \cdot \omega\left(f ; h \cdot \sqrt{1-x^{2}}\right) .
$$

An upper bound for the constant $c$ in Theorem A will now be given using the classical Fejér-Korovkin kernels. Much information on these important kernels is contained in an excellent survey paper by Stark [16]. Their significance arises from the fact that for them the quantity $1-\rho_{1, m(n)}$ is minimised in the following sense.

Let $S_{n}$ be the set of all trigonometric polynomials $T_{n}(t)=1 / 2+\sum_{k=1}^{n} \rho_{k, n} \cos k t$. Set $S_{n}^{+}:=\left\{T_{n} \mid T_{n} \in S_{n}\right.$ and $T_{n}(t) \geqslant 0$ for all $\left.t\right\}$.

Then (see, for example DeVore [5, Theorem 4.2])

$$
\max _{T_{n} \in S_{n}^{+}} \rho_{1, n}\left(T_{n}\right)=\cos \frac{\pi}{n+2}
$$

or

$$
\min _{T_{n} \in S_{n}^{+}}\left(1-\rho_{1, n}\left(T_{n}\right)\right)=1-\cos \frac{\pi}{n+2} .
$$

For the Féjer-Korovkin kernels one has the representation

$$
K_{n}(v):=\frac{1}{n+2}\left(\frac{\sin \pi /(n+2) \cos (n+2) v / 2}{\cos v-\cos \pi /(n+2)}\right)^{2}=\frac{1}{2}+\sum_{k=1}^{n} \rho_{k, n} \cos k v \geqslant 0
$$


and for these we have $\rho_{1, n}=\cos \pi /(n+2)>0$.

In the sequel we shall use operators $G_{m(n)}$ as given in (2.1) and which are defined using the Fejér-Korovkin kernels $K_{n}$. We shall denote these operators by $W_{n}$ and their Boolean sum modifications by $W_{n}^{+}$. We thus have

$$
\begin{aligned}
W_{n}^{+}(f, x)= & W_{n}(f ; x)+\frac{1}{2}(x+1)\left[f(1)-W_{n}(f, 1)\right] \\
& +\frac{1}{2}(1-x)\left[f(-1)-W_{n}(f,-1)\right]
\end{aligned}
$$

where

$$
W_{n}(f ; x)=\frac{1}{\pi} \cdot \int_{-\pi}^{\pi} f(\cos (\arccos x+v)) \cdot K_{n}(v) d v
$$

Our first result is the following

TheOREM 2.6 .

$$
\left|f(x)-W_{n}^{+}(f, x)\right| \leqslant 12 \cdot \omega\left(f, \frac{\sqrt{1-x^{2}}}{n+2}\right) .
$$

Furthermore, if $\omega(f, \cdot)$ is concave, we have

$$
\left|f(x)-W_{n}^{+}(f, x)\right| \leqslant 10 \cdot \omega\left(f, \frac{\sqrt{1-x^{2}}}{n+2}\right) .
$$

Proof: We first observe that for the Fejér-Korovkin kernels we have

$$
\sqrt{1-\rho_{1, n}}=\sqrt{1-\cos \frac{\pi}{n+2}}=\sqrt{2 \sin ^{2} \frac{\pi}{2(n+2)}} \leqslant \sqrt{\frac{2 \pi^{2}}{4(n+2)^{2}}}=\frac{\pi}{\sqrt{2}} \cdot \frac{1}{n+2} .
$$

Choosing $h=1 /(n+2)$ in (2.22) of Theorem 2.4 gives

$$
\begin{aligned}
\left|f(x)-W_{n}^{+}(f, x)\right| & \leqslant\left[2+(3+\sqrt{2}) \cdot \frac{\pi}{\sqrt{2}} \cdot \frac{1}{n+2} \cdot(n+2)\right] \cdot \omega\left(f, \frac{\sqrt{1-x^{2}}}{n+2}\right) \\
& =\left[2+\left(\frac{3}{\sqrt{2}}+1\right) \pi\right] \cdot \omega\left(f, \frac{\sqrt{1-x^{2}}}{n+2}\right) \\
& \leqslant 12 \cdot \omega\left(f, \frac{\sqrt{1-x^{2}}}{n+2}\right) .
\end{aligned}
$$


If $\omega(f, \cdot)$ is concave, we may use $(2.26)$ of Remark 2.5 to obtain

$$
\begin{aligned}
& \left|f(x)-W_{n}^{+}(f, x)\right| \leqslant \max \left[2,(3+\sqrt{2}) \cdot \frac{\pi}{\sqrt{2}} \cdot \frac{1}{n+2} \cdot(n+2)\right] \cdot \omega\left(f, \frac{\sqrt{1-x^{2}}}{n+2}\right) \\
& \leqslant \max \left[2,\left(\frac{3}{\sqrt{2}}+1\right) \pi\right] \cdot \omega\left(f, \frac{\sqrt{1-x^{2}}}{n+2}\right) \\
& \leqslant 10 \cdot \omega\left(f, \frac{\sqrt{1-x^{2}}}{n+2}\right) \text {. }
\end{aligned}
$$

A second parameter frequently used in the modulus is $\pi /(n+1) \sqrt{1-x^{2}}$. This is dealt with in the following theorem:

THEOREM 2.7. For the operators $W_{n}^{+}$we also have the following inequalities:

$$
\left|f(x)-W_{n}^{+}(f, x)\right| \leqslant 6 \cdot \omega\left(f ; \frac{\pi}{n+1} \sqrt{1-x^{2}}\right) .
$$

If $\omega(f, \cdot)$ is concave, one has

$$
\left|f(x)-W_{n}^{+}(f, x)\right| \leqslant 4 \cdot \omega\left(f ; \frac{\pi}{n+1} \sqrt{1-x^{2}}\right) .
$$

Proof: Úsing again $\sqrt{1-\rho_{1, n}} \leqslant \pi / \sqrt{2} \cdot 1 /(n+2)$, and putting $h=\pi /(n+1)$ in (2.22) of Theorem 2.4, gives

$$
\begin{aligned}
\left|f(x)-W_{n}^{+}(f, x)\right| & \leqslant\left[2+(3+\sqrt{2}) \cdot \frac{\pi}{\sqrt{2}} \cdot \frac{1}{n+2} \cdot \frac{n+1}{\pi}\right] \cdot \omega\left(f ; \frac{\pi}{n+1} \sqrt{1-x^{2}}\right) \\
& \leqslant\left[2+\frac{3}{\sqrt{2}}+1\right] \omega\left(f ; \frac{\pi}{n+1} \sqrt{1-x^{2}}\right) \\
& \leqslant 5.13 \cdot \omega\left(f ; \frac{\pi}{n+1} \sqrt{1-x^{2}}\right) \\
& \leqslant 6 \cdot \omega\left(f ; \frac{\pi}{n+1} \sqrt{1-x^{2}}\right)
\end{aligned}
$$

which is (i). If $\omega(f, \cdot)$ is concave, we again use (2.26) from Remark 2.5 to arrive at

$$
\begin{aligned}
\left|f(x)-W_{n}^{+}(f, x)\right| & \leqslant \max \left\{2,(3+\sqrt{2}) \cdot \frac{\pi}{\sqrt{2}} \cdot \frac{1}{n+2} \cdot \frac{n+1}{\pi}\right\} \cdot \omega\left(f ; \frac{\pi}{n+1} \sqrt{1-x^{2}}\right) \\
& \leqslant \max \left\{2,\left(\frac{3}{\sqrt{2}}+1\right)\right\} \cdot \omega\left(f ; \frac{\pi}{n+1} \sqrt{1-x^{2}}\right) \\
& \leqslant 3.13 \cdot \omega\left(f ; \frac{\pi}{n+1} \sqrt{1-x^{2}}\right) \\
& \leqslant 4 \cdot \omega\left(f ; \frac{\pi}{n+1} \sqrt{1-x^{2}}\right) .
\end{aligned}
$$




\section{CONCLUDing REMARKS}

The inequalities of Theorems 2.6 and 2.7 raise the question of how good the constants given there are in comparison to the best possible ones (for algebraic polynomial approximation in general, and not just for the operators $W_{n}^{+}$used in the above). An indication is given in this section. The reader should note, however, that the result of Corollary 3.2 is pointwise and asymptotic in nature, and thus does not imply uniform and general ones such as those from the two theorems mentioned. It is hence the intention of this section to only give a hint as to what might be possible to achieve. Details will become clear from our discussion below.

Let us first recall the definition of the classes $H^{\omega}[-1,1]$, where $\omega$ is a general modulus of continuity. (See, for example, Chapter 7 in [7] for a detailed discussion.) We define

$$
H^{\omega}[-1,1]:=\{f \mid f \in C[-1,1] \text { and } \omega(f, \delta) \leqslant \omega(\delta)\} .
$$

The following result is taken from a paper by Korneičuk and Polovina [10], see Theorem 1 and Remark 4 there.

THEOREM 3.1. Let $\omega(t)$ be an arbitrary concave modulus of continuity. Then for each function $f \in H^{\omega}$ there exists a sequence of algebraic polynomials $P_{n}=P_{n}(f, \cdot)$ of degree $n \in \mathbb{N}_{0}$ such that

$$
\left|f(x)-P_{n}(x)\right| \leqslant \frac{1}{2} \omega\left(\frac{\pi}{n+1} \cdot \sqrt{1-x^{2}}\right)+o\left(\omega\left(\frac{1}{n+1}\right)\right),
$$

holds uniformly for $-1 \leqslant x \leqslant 1$ and for every $f \in H^{\omega}$, and the constant $1 / 2$ is best possible.

The fact that inequality (3.1) indeed holds uniformly for every $f \in H^{\omega}$ can be verified directly from their proof.

From Theorem 3.1 we derive the following pointwise consequence.

COROLlary 3.2. Let $\omega(t)>0, t>0$, be a concave modulus of continuity. Let $f \in H^{\omega}[-1,1]$. Then there is a sequence of algebraic polynomials $P_{n}=P_{n}(f, \cdot)$ of degree $n \in \mathbb{N}_{\text {o }}$ such that

(i)

$$
\varlimsup_{n \rightarrow \infty} \frac{\left|f(x)-P_{n}(x)\right|}{\omega\left(\frac{\pi}{n+1} \cdot \sqrt{1-x^{2}}\right)} \leqslant \frac{1}{2} \text { for each fixed }|x|<1 .
$$

Furthermore, putting $\varepsilon_{n}^{1}(f):=\left|f(-1)-P_{n}(-1)\right| / \omega(1 /(n+1))$ and $\varepsilon_{n}^{2}(f)$ $=\left|f(1)-P_{n}(1)\right| / \omega(1 /(n+1))$ one has

$$
\lim _{n \rightarrow \infty} \varepsilon_{n}^{1}(f)=\lim _{n \rightarrow \infty} \varepsilon_{n}^{2}(f)=0 \text { uniformly for } f \in H^{\omega}[-1,1] .
$$


(ii) $\varlimsup_{n \rightarrow \infty} \frac{\left\|f-P_{n}\right\|}{\omega\left(\frac{\pi}{n+1}\right)} \leqslant \frac{1}{2}$.

ProOF: Since (3.1) of Theorem 3.1 holds uniformly for $|x| \leqslant 1$ and for $f \in$ $H^{\boldsymbol{\omega}}[-1,1]$, for $\varepsilon>0$ we get that there is a natural number $N_{1}\left(N_{1}\right.$ being independent of $|x| \leqslant 1$ and of $f$ ), such that, when $n \geqslant N_{1}$, we have

$$
\left|f(x)-P_{n}(x)\right| \leqslant \frac{1}{2} \omega\left(\frac{\pi}{n+1} \cdot \sqrt{1-x^{2}}\right)+\varepsilon \cdot \omega\left(\frac{1}{n+1}\right) .
$$

Letting $x=-1$ and $x=+1$, we get

$$
\left|f(-1)-P_{n}(-1)\right| \leqslant \varepsilon \cdot \omega\left(\frac{1}{n+1}\right), \text { and }\left|f(1)-P_{n}(1)\right| \leqslant \varepsilon \cdot \omega\left(\frac{1}{n+1}\right)
$$

so that

$$
\varepsilon_{n}^{1}(f):=\frac{\left|f(-1)-P_{n}(-1)\right|}{\omega(1 /(n+1))} \leqslant \varepsilon \text { for all } n \geqslant N_{1}
$$

as well as

$$
\varepsilon_{n}^{2}(f):=\frac{\left|f(1)-P_{n}(1)\right|}{\omega(1 /(n+1))} \leqslant \varepsilon \text { for all } n \geqslant N_{1}
$$

Hence

$$
\lim _{n \rightarrow \infty} \varepsilon_{n}^{1}(f)=\lim _{n \rightarrow \infty} \varepsilon_{n}^{2}(f)=0 \text { uniformly for } f \in H^{\omega}[-1,1] .
$$

Furthermore, from (3.4) we have uniformly for $|x|<1$ that

$$
\frac{\left|f(x)-P_{n}(x)\right|}{\omega\left(\frac{\pi}{n+1} \cdot \sqrt{1-x^{2}}\right)} \leqslant \frac{1}{2}+\frac{\varepsilon \cdot \omega\left(\frac{1}{n+1}\right)}{\omega\left(\frac{\pi}{n+1} \cdot \sqrt{1-x^{2}}\right)} \text { for all } n \geqslant N_{1} .
$$

Using $\omega(f, \lambda \delta) \leqslant(\lambda+1) \omega(f, \delta)$ for $\delta \geqslant 0$ and $\lambda>0$, we get

$$
\omega\left(\frac{1}{n+1}\right)=\omega\left(\frac{\pi \sqrt{1-x^{2}}}{n+1} \cdot \frac{1}{\pi \sqrt{1-x^{2}}}\right) \leqslant\left(\frac{1}{\pi \sqrt{1-x^{2}}}+1\right) \omega\left(\frac{\pi \sqrt{1-x^{2}}}{n+1}\right) .
$$

Hence

$$
\frac{\varepsilon \cdot \omega\left(\frac{1}{n+1}\right)}{\omega\left(\frac{\pi}{n+1} \cdot \sqrt{1-x^{2}}\right)} \leqslant \varepsilon \cdot\left(\frac{1}{\pi \sqrt{1-x^{2}}}+1\right)=\frac{\varepsilon}{\pi \sqrt{1-x^{2}}}+\varepsilon
$$


For an arbitrary $\varepsilon_{1}>0$, taking $0<\varepsilon=\varepsilon_{1} \cdot \sqrt{1-x^{2}},\left(|x|<1\right.$, and hence $\left.\varepsilon \leqslant \varepsilon_{1}\right)$, there is a natural number $N_{2}(x)$ independent of $f$, so that, when $n \geqslant N_{2}(x)$, the inequality

$$
\frac{\varepsilon \cdot \omega\left(\frac{1}{n+1}\right)}{\omega\left(\frac{\pi}{n+1} \cdot \sqrt{1-x^{2}}\right)} \leqslant \frac{\varepsilon_{1}}{\pi}+\varepsilon_{1} \leqslant 2 \cdot \varepsilon_{1}
$$

holds.

From (3.5) we have

$$
\frac{\left|f(x)-P_{n}(x)\right|}{\omega\left(\frac{\pi}{n+1} \cdot \sqrt{1-x^{2}}\right)} \leqslant \frac{1}{2}+2 \cdot \varepsilon_{1}
$$

and thus

$$
\varlimsup_{n \rightarrow \infty} \frac{\left|f(x)-P_{n}(x)\right|}{\omega\left(\frac{\pi}{n+1} \cdot \sqrt{1-x^{2}}\right)} \leqslant \frac{1}{2}+2 \cdot \varepsilon_{1} .
$$

Letting $\varepsilon_{1}$ tend to zero shows the validity of

$$
\varlimsup_{n \rightarrow \infty} \frac{\left|f(x)-P_{n}(x)\right|}{\omega\left(\frac{\pi}{n+1} \cdot \sqrt{1-x^{2}}\right)} \leqslant \frac{1}{2} .
$$

From (3.4) we have

$$
\left\|f-P_{n}\right\| \leqslant \frac{1}{2} \cdot \omega\left(\frac{\pi}{n+1}\right)+\varepsilon \cdot \omega\left(\frac{1}{n+1}\right) \text { for } n \geqslant N_{1} .
$$

Similarly it can be proved that

$$
\varlimsup_{n \rightarrow \infty} \frac{\left\|f-P_{n}\right\|}{\omega\left(\frac{\pi}{n+1}\right)} \leqslant \frac{1}{2} .
$$

REMARK 3.3. The operators yielding the polynomials $P_{n}(x)=P_{n}(f ; x)$ are nonlinear, see $[\mathbf{1 0}]$.

Corollary 3.4. Let $\omega(t)$ be an arbitaray concave modulus of continuity. Then for each function $f \in H^{\omega}[-1,1]$ there exists a sequence of algebraic polynomials $P_{n}=P_{n}(f, \cdot)$ of degree $n \in \mathbb{N}_{0}$ so that

$$
\lim _{n \rightarrow \infty} \frac{\sup \left\{\left\|f-P_{n}(f, \cdot)\right\| ; f \in H^{\omega}[-1,1]\right\}}{\omega\left(\frac{\pi}{n+1}\right)}=\frac{1}{2} .
$$


PROOF: From Theorem 3.1 we get

$$
\sup \left\{\left\|f-P_{n}(f ; \cdot)\right\| ; f \in H^{\omega}[-1,1]\right\} \leqslant \frac{1}{2} \cdot \omega\left(\frac{\pi}{n+1}\right)+o\left(\omega\left(\frac{1}{n+1}\right)\right) .
$$

Let $E_{n}(f)_{C[-1,1]}$ denote the approximation constant for $f$ when approximating by algebraic polynomials of degree $\leqslant n$. Polovina [15] (see also [10]) proved that

$$
\begin{aligned}
\sup \left\{E_{n-1}(f)_{C[-1,1]}: f \in H^{\omega}[-1,1]\right\} \\
=\frac{1}{2} \omega\left(\frac{\pi}{n}\right)-\varepsilon_{n} \omega\left(\frac{\pi}{n}\right), \text { where } 0 \leqslant \varepsilon_{n}=O\left(\frac{1}{\ln n}\right) .
\end{aligned}
$$

Hence

$$
\sup \left\{E_{n}(f)_{C[-1,1]}: f \in H^{\omega}[-1,1]\right\}=\frac{1}{2} \omega\left(\frac{\pi}{n+1}\right)+o\left(\omega\left(\frac{\pi}{n+1}\right)\right) .
$$

Since $E_{n}(f)_{C[-1,1]} \leqslant\left\|f-P_{n}(f ; \cdot)\right\|$, from (3.7) we get

$$
\begin{aligned}
\sup \left\{E_{n}(f)_{C[-1,1]}: f \in H^{\omega}[-1,1]\right\} & \leqslant \sup \left\{\left\|f-P_{n}(f ; \cdot)\right\|: f \in H^{\omega}[-1,1]\right\} \\
& \leqslant \frac{1}{2} \cdot \omega\left(\frac{\pi}{n+1}\right)+o\left(\omega\left(\frac{1}{n+1}\right)\right),
\end{aligned}
$$

and hence

$$
\sup \left\{\left\|f-P_{n}(f ; \cdot)\right\|: f \in H^{\omega}[-1,1]\right\}=\frac{1}{2} \cdot \omega\left(\frac{\pi}{n+1}\right)+o\left(\omega\left(\frac{1}{n+1}\right)\right),
$$

from which it follows that

$$
\frac{\sup \left\{\mid f-P_{n}(f ; \cdot) \|: f \in H^{\omega}[-1,1]\right\}}{\omega\left(\frac{\pi}{n+1}\right)}=\frac{1}{2}+o(1) .
$$

The latter relation implies the statement of Corollary 3.4.

We have thus seen that the asymptotic constant $1 / 2$ is best possible in an equality like that of Corollary 3.4.

Comparing the assertions of Theorem 2.7 (ii) and of Corollary 3.2 shows that there appears to be room for improvement on the constant 4 figuring in (2.27). A guideline for future research may be another result of Korneičuk. In his book [7] (see also [9]) it is shown that for each function $f \in C_{2 \pi}, f \neq$ constant, one has

$$
E_{n}(f)_{C_{2 \pi}}<1 \cdot \omega\left(f, \frac{\pi}{n}\right), n \in \mathbb{N}
$$

where the constant 1 is best possible. Here $E_{n}(f)_{C_{2 \pi}}$ is the best uniform approximation of $f$ by trigonometric polynomials of degree $\leqslant n-1$.

The results of Corollaries 3.2 and 3.4, together with above remark concerning the trigonometric case, suggest the following 
Conjecture 3.5. For each function $f \in C[-1,1], f \neq$ constant, and $n \geqslant 1$, there is an algebraic polynomial $P_{n}(x)$ of degree $\leqslant n$ such that

$$
\left|f(x)-P_{n}(x)\right|<1 \cdot \omega\left(f, \frac{\pi \sqrt{1-x^{2}}}{n+1}\right) \text { for all }|x|<1
$$

where the constant 1 is best possible.

\section{REFERENCES}

[1] J-D. Cao, 'Generalizations of Timan theorem, Lehnhoff theorem and Telyakovskiị theorem', (Chinese), Kexue Tongbao 15 (1986), 1132-1135; (English translation Science Bulletin (English Edition) 32 (1987), 1225-1229).

[2] J-D. Cao, 'Generalization of Timan's theorem, Lehnhoff's theorem and Telyakovskii's theorem', in Schriftenreihe des Fachbereichs Mathematik SM-DU-106 (Universität Duisburg, Germany, 1986).

[3] J-D. Cao and H.H. Gonska, 'Approximation by Boolean sums of linear operators: Telyakovskiī-type estimates', Bull. Austral. Math. Soc. 42 (1990), 253-266.

[4] J-D. Cao and H.H. Gonska, 'Pointwise estimates for higher order convexity preserving polynomial approximation', J. Austral. Math. Soc. Ser. B 36 (1994), 213-233.

[5] R.A. DeVore, The Approximation of continuous functions by positive linear operators (Springer-Verlag, Berlin, Heidelberg, New York, 1972).

[6] V.K. Dzjadyk, Introduction to the theory of uniform approximation of functions by polynomials, (Russian) (Izdatel'stvo Nauka, Moscow, 1977).

[7] N.P. Korneičuk, Extremal problems of approximation theory, (Russian) (Izdatel'stvo Nauka, Moscow, 1976).

[8] N.P. Korneičuk, 'On best constants in Jackson's inequality for continuous periodic functions', (Russian), Mat. Zametki 32 (1982), 669-674.

[9] N.P. Korneičuk, Exact constants in approximation theory (Cambridge University Press, Cambridge, 1991).

[10] N.P. Korneičuk and A.I. Polovina, 'Approximation of continuous functions by algebraic polynomials', (Russian), Ukrain. Math. J. 24 (1972), 328-340.

[11] H.G. Lehnhoff, 'A simple proof of A.F. Timan's theorem', J. Approx. Theory 38 (1983), 172-176.

[12] H.G. Lehnhoff, 'A new proof of Telyakovskiǐ's theorem', J. Approx. Theory 38 (1983), 177-181.

[13] A. Lupaş, 'On the approximation of continuous functions', Publ. Inst. Math. (Beograd) 40 (56) (1986), 75-83.

[14] S.A. Pičugov, 'Approximation of continuous functions on a segment by linear methods', (Russian), Mat. Zametki 24 (1978), 343-348.

[15] A.I. Polovina, 'On the best approximation of continuous functions on the interval $[-1,1]$ ', (Ukrainian), Dopovīd̄̄ Akad. Nauk Ukraï. RSR Ser. A (1964), 722-726. 
[16] E.L. Stark, 'The kernel of Féjer-Korovkin: a basic tool in the constructive theory of functions', in Functions, Series, Operators I, II, (B. Sz. -Nagy and J. Szabados, Editors), (Proc. Int. Conf. Budapest 1980) (North Holland, Amsterdam, New York, 1983).

[17] S.A. Telyakovskiǐ, 'Two theorems on the approximation of functions by algebraic polynomials', (Russian), Mat. Sb. 70 (1966), 252-265.

\author{
Department of Mathematics \\ Fudan University \\ Shanghai 200433 \\ People's Republic of China
}

\author{
Department of Mathematics \\ University of Duisburg \\ D-47048 Duisburg \\ Germany
}

